\title{
Moderate hypothermia in the management of resistant automatic tachycardias in children
}

\author{
Seshadri Balaji, Ian Sullivan, John Deanfield, Ian James
}

\begin{abstract}
Background-Automatic focus tachycardias are often resistant to electrical and pharmacological treatment. Moderate systemic hypothermia $\left(32-34^{\circ} \mathrm{C}\right)$ may reduce the tachycardia rate in children with His bundle tachycardia after cardiac surgery.

Methods-The case notes of seven children with automatic focus tachycardias treated with hypothermia were reviewed. Six had His bundle tachycardia after cardiac surgery and one had ectopic atrial tachycardia; all had signs of low cardiac output.
\end{abstract}

Results-Hypothermia led to a reduction in heart rate in all patients (from 211 (28) (mean (SD)) to 146 (5) beats/ minute, $\mathrm{p}<0.001$ ), with rises in systolic blood pressure (from 74 (14) $\mathrm{mm} \mathrm{Hg}$ to 97 (10) $\mathrm{mm} \mathrm{Hg}, \mathrm{p}<0.01$ ) and hourly urine output (from $0.5(0.4) \mathrm{ml} / \mathrm{kg}$ to 4.6 (2.8) $\mathrm{ml} / \mathrm{kg}, \mathrm{p}<0.02)$. No direct adverse effects were noted. The arrhythmia did not resolve in three children, who died (two with His bundle tachycardia after Fontan procedures and one with ectopic atrial tachycardia); the other four regained sinus rhythm which was maintained at follow up of 3-13 (mean 9) months.

Conclusions-Moderate systemic hypothermia led to slowing of the arrhythmia rate and an improvement in cardiac output in patients with resistant automatic focus tachycardias. It can be used to improve the haemodynamic condition while other measures of arrhythmia control are being pursued or until spontaneous recovery of normal rhythm.

Accepted for publicatio 29 April 1991
In children with congenital heart disease automatic tachycardia is often resistant to electrical and pharmacological treatment ${ }^{1}$ and may lead to fatal haemodynamic deterioration. ${ }^{12}$ Some automatic tachycardias, notably His bundle tachycardia after cardiac surgery, are self-limiting. ${ }^{3}$ Any measures that might improve the haemodynamic state could be useful in the management of the patient while other means of controlling the tachycardia are being pursued or while spontaneous recovery is awaited.

Hypothermia reduced the automaticity of all the cardiac pacemaker tissues ${ }^{4}$ and it was beneficial in the management of His bundle tachycardia after cardiac surgery. ${ }^{56}$ We report the effect of moderate hypothermia $\left(32^{\circ} \mathrm{C}\right.$ to $34^{\circ} \mathrm{C}$ ) in seven children with resistant automatic tachycardias and congenital heart disease.

\section{Patients and methods}

PATIENTS

Between November 1988 and July 1990 we used moderate hypothermia to treat seven children with resistant tachycardia. Table 1 shows their clinical details.

A 4 day old boy who had undergone balloon atrial septostomy on the third day of life for transposition of the great arteries with a ventricular septal defect developed incessant ectopic atrial tachycardia with a rate of 200 220 beats per minute. Administration of adenosine produced brief atrioventricular block (fig 1). Drug treatment was attempted, initially with digoxin, then with digoxin and propranolol, subsequently with flecainide, and lastly with amiodarone, but the tachycardia rate fell by less than 30 beats per minute. At the age of 20 days he underwent electrophysiological study and a high right atrial focus for the tachycardia was identified. Hypothermia was started when his haemodynamic state deteriorated after an unsuccessful attempt at surgical cryoablation of the focus of the arrhythmia.

The other six patients developed $\mathrm{His}$ bundle tachycardia 2-48 hours after cardiac surgery. Antiarrhythmic drug treatment was started in the first two patients (amiodarone and flecainide respectively in numbers 3 and 7 (table 1)) but it had no effect on the heart rate or the rhythm. Amiodarone was continued as an infusion in patient 3 and flecainide in patient 7 during the period of hypothermia.
Ad, adenosine; Am, amiodarone; APC, atriopulmonary connection; BAS, balloon atria septostomy; DCS, direct current shock; Dig, digoxin; DILV, double inlet left ventricle; EAT ectopic atrial tachycardia; F, Fontan procedure; Fl, flecainide; HBT, His bundle tachycardia MBTS, modified Blalock Taussig shunt; $\mathrm{Pr}$, propranolol; PS, pulmonary stenosis; TA, tricuspid atresia; TOF, tetralogy of Fallot; TGA, transposition of the great arteries; TCPC, total cavopulmonary connection; VSD, ventricular septal defect. 
Figure 1 Continuous electrocardiographic recording (surface lead V1) in a child with ectopic atrial tachycardia (patient 1). Adenosine was given at point $A$ (arrow) and caused transient atrioventricular nodal block which unmasked the atrial tachycardia (small arrows).

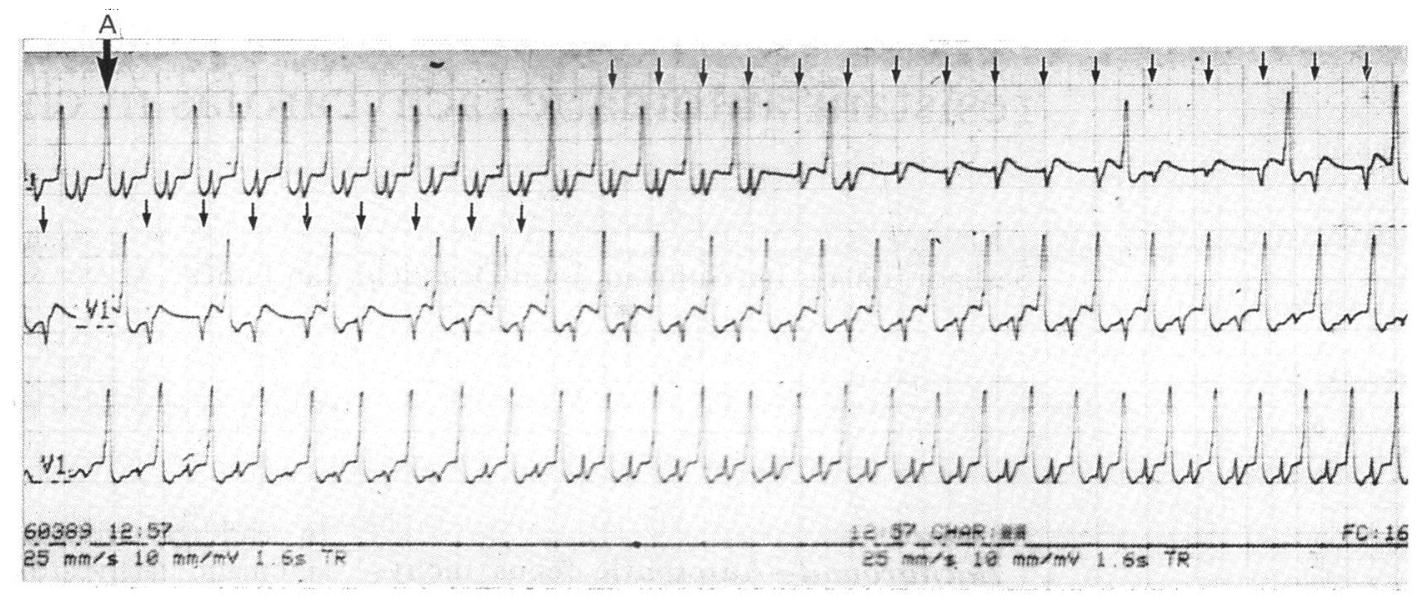

Figure 2

Electrocardiogram (lead II) and blood pressure tracing of a patient with His bundle tachycardia showing a narrow $Q R S$ complex tachycardia with atrioventricular dissociation and considerable beat to beat variability of blood pressure.

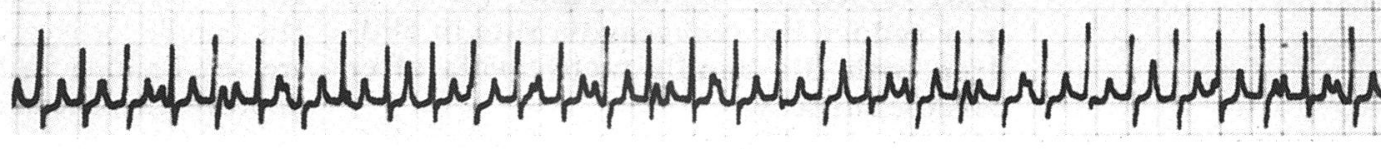
100

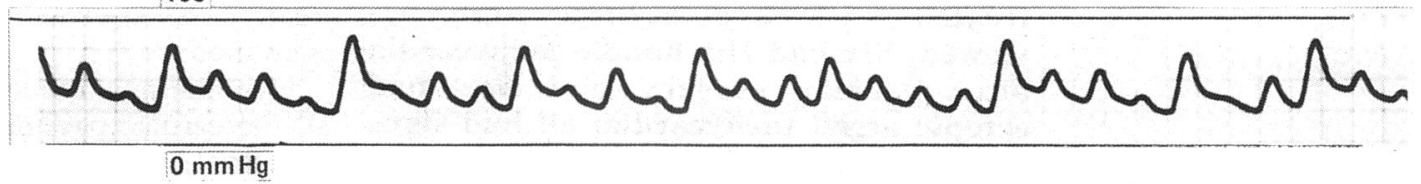

Patient 7 was also treated with digoxin throughout his postoperative course.

After this early experience hypothermia was used as the initial treatment in patients developing His bundle tachycardia after cardiac surgery. However, overdrive pacing was attempted, without success, in one patient (patient 5) before hypothermia was induced.

\section{METHODS}

Arrhythmia diagnosis

The diagnosis of His bundle tachycardia was based on the presence of a regular tachycardia with a QRS complex similar to that in sinus rhythm. Each patient had atrioventricular dissociation and a ventricular rate in excess of the atrial rate (fig 2). Atrial electrograms recorded via the atrial pacing lead inserted at the time of surgery confirmed the diagnosis (fig 3 ). The evidence of beat to beat variability in the arterial pressure trace despite the presence of a regular ventricular rhythm further supported the diagnosis of atrioventricular dissociation (fig 2).

Ectopic atrial tachycardia was diagnosed in a neonate with 1:1 atrioventricular relation and a heart rate which was consistently 200220 beats per minute. The highest heart rate (mean (SD)) in normal neonates in sinus rhythm is 179 (19) beats/minute. ${ }^{7}$ When adenosine induced a transient atrioventricular block the atrial tachycardia was unmasked (fig 1). The diagnosis was confirmed by invasive electrophysiological study.

\section{Hypothermia}

Surface cooling was achieved by placing ice packs around the head and by gastric irrigation with cold water. If this proved inadequate a heat exchanging blanket was placed under the patient to achieve the desired core temperature. The rectal temperature was used as the reference value in all patients. All patients were sedated, paralysed, and on ventilatory support during the period of hypothermia.

Patients were cooled to a temperature of 32$34^{\circ} \mathrm{C}$ until the heart rate fell to 150 beats per minute or less. Once started, cooling was maintained for at least 36 hours. Cooling was stopped when the patient was judged to have been stable for at least 24 hours (on the basis of continuous measurement of blood pressure and urine output and intermittent arterial blood gas analysis). Resolution of the arrhythmia was not a criterion for rewarming.

\section{Results}

ECTOPIC ATRIAL TACHYCARDIA

In the one neonate with this arrhythmia the heart rate fell from 220 to 150 beats per minute

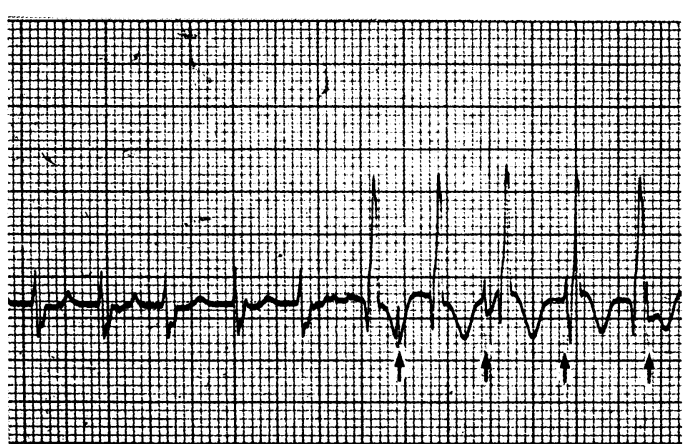

Figure 3 Electrogram recorded from an atrial epicardia pacing wire (right half of the picture) showing His bundle tachycardia in a patient in whom the standard

electrocardiogram (lead II, left) was not diagnostic. The arrows indicate atrial depolarisation. 
Table 2 Data on hypothermia treatment

\begin{tabular}{llllllll}
\hline $\begin{array}{l}\text { Patient } \\
\text { No }\end{array}$ & $\begin{array}{l}\text { Interval } \\
(\boldsymbol{h})\end{array}$ & $\begin{array}{l}\text { HR during } \\
\text { arrhy } \\
\text { (beats } / \text { min) }\end{array}$ & $\begin{array}{l}\text { Central } T^{\circ} \\
\text { during arrhy } \\
\left({ }^{\circ} \mathrm{C}\right)\end{array}$ & $\begin{array}{l}\text { Central } T^{\circ} \\
\text { cooled } \\
\left({ }^{\circ} \mathrm{C}\right)\end{array}$ & $\begin{array}{l}\text { HR when } \\
\text { cooled } \\
\text { (beats/min) }\end{array}$ & $\begin{array}{l}\text { Duration } \\
\text { cooled }\end{array}$ & Outcome \\
\hline 1 & 24 & 220 & 37 & 32 & 150 & 4 days & Died $\dagger$ \\
2 & 2 & 250 & $39 \cdot 5$ & 34 & 150 & 5 days & SR \\
3 & 18 & 230 & 38 & 34 & 150 & $38 \mathrm{~h}$ & SR \\
4 & 2 & 220 & $37 \cdot 5$ & 33 & 140 & $67 \mathrm{~h}$ & SR \\
5 & 48 & 210 & 37 & 34 & 140 & $36 \mathrm{~h}$ & SR \\
6 & 48 & 170 & 39 & 34 & 150 & $48 \mathrm{~h}$ & Died \\
7 & 2 & 180 & 40 & 33 & 140 & $48 \mathrm{~h}$ & Died \\
\hline
\end{tabular}

^Between procedures and onset of arrhythmia.

+ Died at surgery (Senning procedure).
HR, heart rate; $S R$, sinus rhythm; $T^{\circ}$, temperature.

with apparent improvement in cardiac output when cooling to a temperature of $32^{\circ} \mathrm{C}$ was started. Systolic blood pressure improved from 60 to $70 \mathrm{~mm} \mathrm{Hg}$ and hourly urine output, which had been $0.2 \mathrm{ml} / \mathrm{kg}$ for the four hours before hypothermia, improved to $1.6 \mathrm{ml} / \mathrm{kg}$ for the next four hours. He was stable for the next four days. When he was allowed to become normothermic, however, the ectopic atrial tachycardia rate increased to 200 beats/minute and he remained dependent on assisted ventilation and peritoneal dialysis. Eventually, a Senning procedure with closure of ventricular septal defect was undertaken on day 26 in the hope that the extensive atrial surgery might ablate the focus for the tachycardia. Unfortunately he could not be weaned from cardiopulmonary bypass.

\section{HIS BUNDLE TACHYCARDIA}

Six patients had His bundle tachycardia after cardiac surgery. Four of these had surgery in the region of the atrioventricular bundle: repair of tetralogy of Fallot in three and closure of a perimembraneous inlet ventricular septal defect in one. The other two patients had Fontan operations-one an atriopulmonary connection and the other a total cavopulmonary connection.

Conventional antiarrhythmic drug treatment was the initial treatment in two patients (table 1) but it did not restore sinus rhythm or reduce the heart rate to below 180 beats per minute (table 2). Treatment with antiarrhythmic drugs (amiodarone in one and digoxin and flecainide in another) was continued in two patients. Surface cooling reduced the heart rate and improved indirect measures of cardiac output in all cases. Systolic blood pressure improved from 77 (14) $\mathrm{mm} \mathrm{Hg}$ (mean (SD)) at the start of cooling to $97(10) \mathrm{mm} \mathrm{Hg}$ ( $p<0.01$ ) when the target core temperature was reached (fig 4). Hourly urine output improved from $0.5(0.4) \mathrm{ml} / \mathrm{kg}$ for the four hours before hypothermia to $4.6(2.8)$ for the first four hours after the start of hypothermia ( $p$

Figure 4 Effect of hypothermia on systolic blood pressure and urine output in all seven patients. See text for details of measurement.
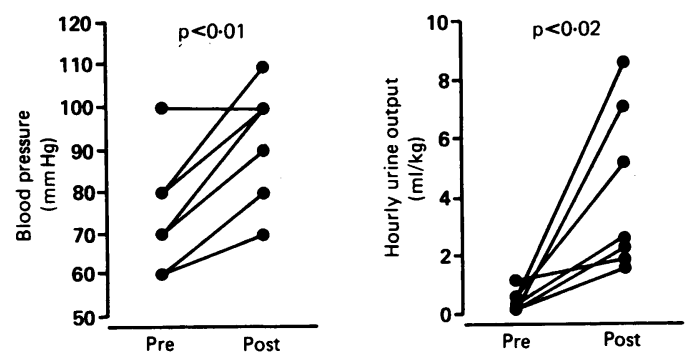

$<0.02$ ) (fig 4). Figure 5 shows the effect of starting hypothermia on the heart rate and urine output in patient 3.

Two children regained sinus rhythm while they were still hypothermic (after four hours in patient 5 and after 66 hours in patient 4). Hypothermia was continued at $34^{\circ} \mathrm{C}$ in patient 5 for a further 32 hours as a precaution against the recurrence of the tachycardia, while patient 4 was rewarmed one hour after sinus rhythm was regained. Two other children (patients 2 and 3) regained sinus rhythm 40 and 84 hours respectively after rewarming. The two children who had undergone Fontan procedures (patients 6 and 7) did not regain sinus rhythm and died soon after rewarming. The four survivors (patients 2, 3, 4, and 5) maintained sinus rhythm during follow up of 3-13 months (mean 9 months).

No patient developed a complication that was thought to be a direct result of hypothermia. None developed additional ventricular arrhythmias. One patient (patient 3) was paced sequentially at 140 beats per minute because of a transient lowering of heart rate to 110 beats per minute. One other (patient 4 ) was sequentially paced at 150 beats per minute for four hours when her heart rate fell to 120 beats per minute. Pacing was stopped because neither blood pressure nor urine output improved. No other patient was electively paced during hypothermia. There was no change in the arterial blood $\mathrm{pH}$ during cooling in any patient (mean $7.38(0.09)$ before cooling and $7.45(0.05)$ during cooling, $p>0.1)$ and none developed metabolic acidosis owing to cooling. One patient (patient 2) had pulmonary hypertensive crises on rewarming. He did not have pulmonary hypertensive episodes before

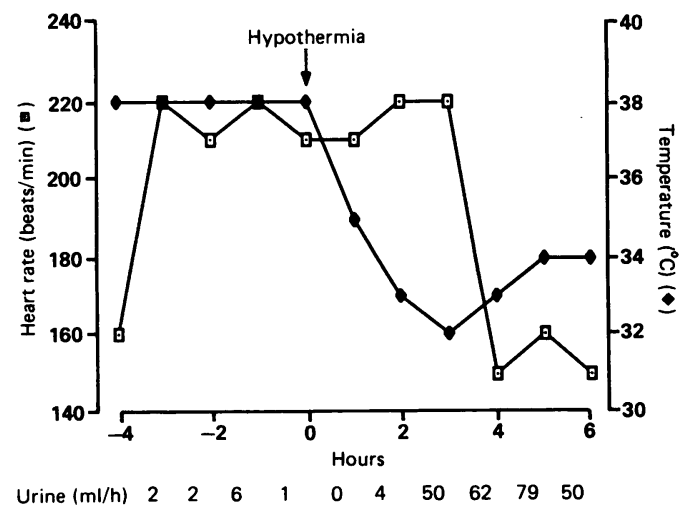

Figure 5 Effect of hypothermia on the heart rate and urine output in patient 3. Heart rate fell to 150 beats per minute when the core temperature reached $32^{\circ} \mathrm{C}$. Urine output improved soon after hypothermia was started. 
or during hypothermia. He also had a chest infection caused by Staphylococcus aureus at the time of rewarming. Rewarming was uneventful in patients 3, 4, and 5. Soon after rewarming patient 3 developed a chest infection caused by Klebsiella which led to ventilatory support being prolonged. Both patients 2 and 3, however, had been on assisted ventilation for eight and six days respectively at the time of diagnosis of the chest infection.

\section{Discussion}

Hypothermia reduces the automaticity of cardiac pacemaker cells and therefore may be valuable in the treatment of ectopic focus tachycardias. Persistent tachycardia may lead to haemodynamic deterioration, particularly in children with congenital heart defects. Though hypothermia in normal subjects is known to reduce cardiac output and increase systemic vascular resistance ${ }^{8}$ it also reduces the oxygen demand so that the oxygen supply-demand balance is unaffected, provided the shivering response to hypothermia has been abolished by the use of paralysis and artificial ventilation.

His bundle tachycardia after heart surgery in children is often self-limiting. ${ }^{3}$ Nevertheless, it is often difficult to control, causes acute haemodynamic instability, and can be fatal. ${ }^{23}$ It is unresponsive to most conventional forms of treatment. ${ }^{39}$ Digoxin, ${ }^{2}$ propafenone, ${ }^{10}$ and flecainide ${ }^{9}$ have been reported to reduce the tachycardia rate to some extent. Both flecainide and propafenone, however, have a negative inotropic effect and there is no ideal drug or electrical treatment.

Bash et al reported the successful use of hypothermia in three infants ${ }^{5}$ and Guccione et al reported it in two. ${ }^{6}$ All had His bundle tachycardia after surgery for congenital heart disease, though none had undergone a Fontan procedure. Before they used a surface cooling blanket to produce systemic hypothermia Bash et al unsuccessfully used ice to the face alone in one case, ice to the face with digoxin and direct current cardioversion in one case, and digoxin and lignocaine in the other. Guccione et al reported that pharmacological treatment was unsuccessful but did not report the specific agents tried.

It has been suggested that $\mathrm{His}$ bundle tachycardia after cardiac surgery in children is unlikely to cause major haemodynamic deterioration at a rate below 200 beats per minute. ${ }^{10}$ This has not been our experience. Loss of sinus rhythm and atrioventricular synchrony is poorly tolerated by patients after a Fontan operation. ${ }^{11}$ His bundle tachycardia, even at rates of about 180 beats per minute, was poorly tolerated by our two patients who had modified Fontan operations. The period of hypothermia was relatively short ( 48 hours) in both. All three infants reported by Bash et al regained sinus rhythm after rewarming after periods of hypothermia ranging from four to 24 hours, ${ }^{5}$ whereas both children reported by Guccione et al did so while still being cooled 12 and 42 hours after the start of hypothermia. Two of our patients regained sinus rhythm during the cooling phase and the other two did so after rewarming. In retrospect, rewarming may have been attempted too early in the two patients who had modified Fontan procedures. A longer duration of cooling may have made the resumption of sinus rhythm more likely. $R$ wave synchronised atrial pacing has been described as an adjunct to hypothermia in the management of postoperative $\mathrm{His}$ bundle tachycardia $^{12}$ and may be especially useful in the management of this condition after Fontan type operations.

In the neonate with ectopic atrial tachycardia, cooling was attempted only after other measures, both invasive and non-invasive, had failed. Hypothermia was successful in producing haemodynamic stability while he was waiting for operation. He never regained sinus rhythm.

Induction of hypothermia is simple and we did not recognise any unwanted side effects. The occurrence of pulmonary hypertensive crises during rewarming in one patient, a 7 month old infant who had undergone repair of a large ventricular septal defect, was thought to be related more to a chest infection than to the rewarming process. We were concerned that there may be a link with infection, which occurred in two of our patients. Both of them, however, had prolonged endotracheal intubation together with artificial ventilation and suboptimal cardiac output. In view of the complexity of their postoperative course we believe that a direct relation between hypothermia and infection cannot be established.

Moderate hypothermia can be used safely to manage children with His bundle tachycardia after cardiac surgery. It seems to slow the arrhythmia rate and so improve haemodynamic state. This may contribute to restoration of sinus rhythm or buy time before the arrhythmia resolves spontaneously. It may also produce similar benefit in other forms of automatic tachycardia and be useful as an adjunct to electrical or pharmacological treatment.

1 Gillette PC. Evolving concepts in the management of congenital junctional ectopic tachycardia. Circulation 1990;81:1713-4.

2 Grant JW, Serwer GA, Armstrong BE, Oldham HN, Anderson PAW. Junctional tachycardia in infants and children after open heart surgery for congenital heart disease. Am J Cardiol 1987;59:1216-8.

3 Gillette PC. Diagnosis and management of post-operative junctional ectopic tachycardia. Am Heart $J$ 1989;118: 192-4.

4 Hicks CE, McCord WC, Blount SG. Electrocardiographic changes during hypothermia and circulatory occlusion Circulation 1956;13:21-8.

5 Bash SE, Shah JJ, Albers WH, Geiss DM. Hypothermia for the treatment of post surgical greatly accelerated junctional ectopic tachycardia. J Am Coll Cardiol 1987 10:1095-9.

6 Guccione P, Papa M, Pasquini L, et al. Junctional ectopic tachycardia after paediatric cardiac surgery; successfu treatment with hypothermia [abstract]. Eur Heart 1989;10:105.

7 Southall DP, Richards J, Mitchell P, Brown DJ, Johnston PGB, Shinebourne EA. Study of cardiac rhythm in healthy newborn infants. Br Heart J 1980;43:14-20.

8 Sealy WC. Hypothermia. Its possible role in cardiac surgery. Ann Thorac Surg 1989;47:788-91.

9 Wren C, Campbell RWF. His bundle tachycardia - arrhyth mogenic and antiarrhythmic effects of therapy. Eur Heart 1987;8:647-50.

10 Garson A, Moak JP, Smith RT, Norton JB. Usefulness of intravenous Propafenone for control of post-operative
junctional ectopic tachycardia. Am J Cardiol 1987; 59:1422-4.

11 Weber HS, Hellenbrand WE, Kleinman CS, et al. Predictors of rhythm disturbances and subsequent morbidity after the Fontan operation. Am J Cardiol 1989;64:762-7.

12 Till JA, Rigby ML, Rowland E. R Wave synchronised atrial pacing as an adjunct to the management of His bundle tachycardia
1990;64:91. 